\title{
Validating ocean tide loading models using GPS
}

\author{
C. Urschl, R. Dach, U. Hugentobler, S. Schaer, G. Beutler \\ Astronomical Institute, University of Berne, Sidlerstrasse 5, 3012 Bern, Switzerland \\ e-mail: claudia.urschl@aiub.unibe.ch \\ Received:28 October 2003/ Accepted: 26 October 2004 / Published online: 23 February 2005
}

\begin{abstract}
Ocean tides cause periodic deformations of the Earth's surface, also referred to as ocean tide loading (OTL). Tide-induced displacements of the Earth's crust relying on OTL models are usually taken into account in GPS (Global Positioning System) data analyses. On the other hand, it is also possible to validate OTL models using GPS analyses. The following simple approach is used to validate OTL models. Based on a particular model, instantaneous corrections of the site coordinates due to OTL are computed. Site-specific scale factors, $f$, for these corrections are estimated in a standard least-squares adjustment process of GPS observations together with other relevant parameters. A resulting value of $f$ close to unity indicates a good agreement of the model with the actual site displacements. Such scale factors are computed for about 140 globally distributed IGS (International GPS Service) tracking sites. Three OTL models derived from the ocean tide models FES95.2.1, FES99, and GOT00.2 are analyzed. As expected, the most reliable factors are estimated for sites with a large loading effect. In general, the scaling factors have a value close to unity and no significant differences between the three ocean tide models could be observed. It is found that the validation approach is easy to apply. Without requiring much additional effort for a global and self-consistent GPS data analysis, it allows detection of general model misfits on the basis of a large number of globally distributed sites. For detailed validation studies on OTL models, the simultaneous estimation of amplitudes and phases for the main contributing partial tides within a GPS parameter adjustment process would provide more detailed answers.
\end{abstract}

Key words: Ocean tide loading - Ocean tide models Global Positioning System

Correspondence to: C. Urschl

\section{Introduction}

Tidal forces due to the Moon and Sun deform the Earth and are responsible for solid-Earth tides and ocean tides. They produce temporal variations of the Earth's gravity field and measurable periodic deformations of the Earth's surface. Site displacements caused by solidEarth tides may reach amplitudes of up to $40 \mathrm{~cm}$ (Vaníček and Krakiwsky 1986). In addition, ocean tides induce a temporal variation of the ocean mass distribution. The associated periodic deformation of the Earth's crust is known as ocean tide loading (OTL).

The vertical site displacements due to OTL may reach values of several centimeters for coastal sites, and they may be measured using space geodetic techniques such as the Global Positioning System (GPS) (see e.g. Baker et al. 1995). For a baseline with a length of $100 \mathrm{~km}$, directed inland from the coast, the relative OTL effect may reach a magnitude of $1 \mathrm{~cm}$ and should be taken into account not only for undifferenced but also for interferometric processing of GPS data (Dach and Dietrich 2001).

In high-precision GPS data analyses, OTL models are usually applied to account for these displacements of the Earth's surface. However, it is also possible to derive OTL effects from site displacements measured with GPS tracking data. The displacement of sites due to OTL may be described as a sum of harmonic oscillations. The frequencies of the main constituents are well known because the loading signal consists of the same partial tides and frequencies as the tide-generating potential. Based on the frequency content, site-specific amplitudes and phases of the corresponding constituents may be obtained from GPS measurements.

There are several previous studies on deriving OTL parameters from vertical and horizontal position time series estimated from GPS tracking data within regional networks (see e.g. Khan and Scherneck 2002; Kirchner 2001). The most appropriate approach, however, would be to solve for site-specific OTL parameters (amplitudes and phases) in a global GPS data analysis, including 
other relevant parameters. However, the resulting large number of parameters to be estimated in such a solution imposes high demands on computational resources. For each site, six OTL parameters (three components each of amplitude and phase) for each partial tide have to be set up in the parameter adjustment process. Taking into account the 11 main tidal constituents, 9900 parameters have to be determined for a global network of about 150 GPS tracking sites, in addition to station coordinates, troposphere, orbit, Earth orientation, and phase ambiguity parameters. Schenewerk et al. (2001) have done such an analysis with some restrictions to decrease the number of parameters. They estimated 16 OTL parameters for each site, the vertical component of amplitudes and phases of eight main tidal terms using Helmert blocking technique to cope with the large number of parameters.

Our study focuses on a more simplified method of validating selected OTL models on the basis of GPS measurements. We estimate only one additional parameter, a scaling factor, for each site of a global GPS tracking network. We accept the loss of site-specific amplitude and phase information in exchange for a relatively small number of parameters. Nevertheless, we can obtain general validation information for global OTL models.

The aim of this paper is to present validation results obtained with this simple approach. Section 2 gives a short overview of the three ocean tide models considered, FES95.2.1, FES99, and GOT00.2. Section 3 explains the OTL effect. The validation method is demonstrated in Sect. 4. Section 5 presents the data set and the GPS processing strategy used for validation, and Sect. 6 analyzes the validation results and discusses the estimated scaling factors for the global solution, as well as for a few selected GPS sites.

\section{Ocean tide models}

Ocean tide models give the variations in ocean surface height due to tide-generating forces for each geographical location in the ocean and for each point in time. Most of the ocean tide models provide amplitudes and phases for the 11 main tidal constituents: the semidiurnal waves $M_{2}, S_{2}, N_{2}, K_{2}$, the diurnal waves $K_{1}, O_{1}$, $P_{1}, Q_{1}$, and the long-period waves $M_{f}, M_{m}$ and $S_{s a}$. More than $95 \%$ of the tidal signal is characterized by these 11 constituents (Lambeck 1988).

Several ocean tide models are available, varying in characteristics such as the methods of their determination, additionally used empirical tide information, and spatial resolution. The ocean tide models considered in this paper are the Grenoble tide model, FES95.2.1 (Le Provost et al. 1998), the LEGOS tide model, FES99 (Lefèvre et al. 2002), and the Goddard tide model, GOT00.2 (Ray 1999; Petrov and Ma 2002). All of them are based on the pure hydrodynamic model FES94 (Le Provost et al. 1994) as a reference model. FES95.2.1 is a hydrodynamic tide model taking into account TOPEX/ Poseidon altimeter data. It is given on a $0.5 \times 0.5$ degree grid. The more recent models FES99 and GOT00.2, recommended by the IERS Conventions 2000 (McCarthy 2003), are also hydrodynamic models using TOPEX/ Poseidon data. FES99, in addition, assimilates tidegauge observations and provides a $0.25 \times 0.25$ degree resolution. GOT00.2, with a $0.5 \times 0.5$ degree grid, additionally utilizes ERS1/2 altimeter data.

The accuracy of these models depends very much on the TOPEX/Poseidon altimetry data, and is thus limited by the restricted TOPEX/Poseidon coverage in latitude of $\pm 66^{\circ}$. The tide model GOT00.2 is different from the FES tide models in polar regions because ERS1/2 altimetry measurements are used with a coverage in latitude of about $\pm 82^{\circ}$.

The quality of the computed OTL deformations depends on the ocean tide model used. Shum et al. (1997) have shown that most of the global ocean tide models agree to within $2-3 \mathrm{~cm}$ in the deep ocean. The largest differences between the models occur in shallow waters and coastal seas, indicating that these areas are not accurately mapped by global ocean tide models. The spatial resolution of global tide models is, in general, only of the order of $50 \mathrm{~km}$, and significantly limits the accuracy near coastal regions.

\section{Ocean tide loading (OTL) models}

Deformations due to OTL can be derived from ocean tide models by an integration over all global water masses using a Green's function. This function describes the elastic response of the Earth's crust to the surface load (see e.g. Farrell 1972; Scherneck 1991).

The OTL values considered in this study are those computed by Scherneck (2003). The site-specific amplitudes and phases of the horizontal and vertical loading displacements may be extracted for the 11 main constituents for each ocean tide model. The Green's functions for the Gutenberg-Bullen Earth model are used to determine the deformation of the Earth's crust due to point loads. The coastline information is taken from the GMT (Generic Mapping Tools) software package (Wessel and Smith 2004) with a resolution of about $0.6 \mathrm{~km}$.

The displacement vector, e, due to OTL at time $t$ can be expressed as a sum over $n$ contributing individual ocean tides and is given in vertical, north-south, and east-west components (see e.g. Seidelmann 1992)

$\mathbf{e}(t)=\sum_{i=1}^{n}\left(\begin{array}{cc}A_{i}^{\mathrm{V}} & \cos \left(\omega_{i} t+\phi_{i}-\delta_{i}^{\mathrm{v}}\right) \\ A_{i}^{\mathrm{NS}} & \cos \left(\omega_{i} t+\phi_{i}-\delta_{i}^{\mathrm{NS}}\right) \\ A_{i}^{\mathrm{EW}} & \cos \left(\omega_{i} t+\phi_{i}-\delta_{i}^{\mathrm{EW}}\right)\end{array}\right)$

where $\omega_{i}$ is the frequency of the tidal constituents and $\phi_{i}$ the corresponding astronomical argument. The sitespecific amplitudes $A_{i}^{\mathrm{V}}, A_{i}^{\mathrm{NS}}, A_{i}^{\mathrm{EW}}$ and Greenwich phase lags $\delta_{i}^{\mathrm{v}}, \delta_{i}^{\mathrm{NS}}, \delta_{i}^{\mathrm{EW}}$ of each tidal constituent are provided by the particular OTL model for each position requested.

In this study, we compute the OTL effect caused by the 11 main tidal constituents $(n=11$, provided by 


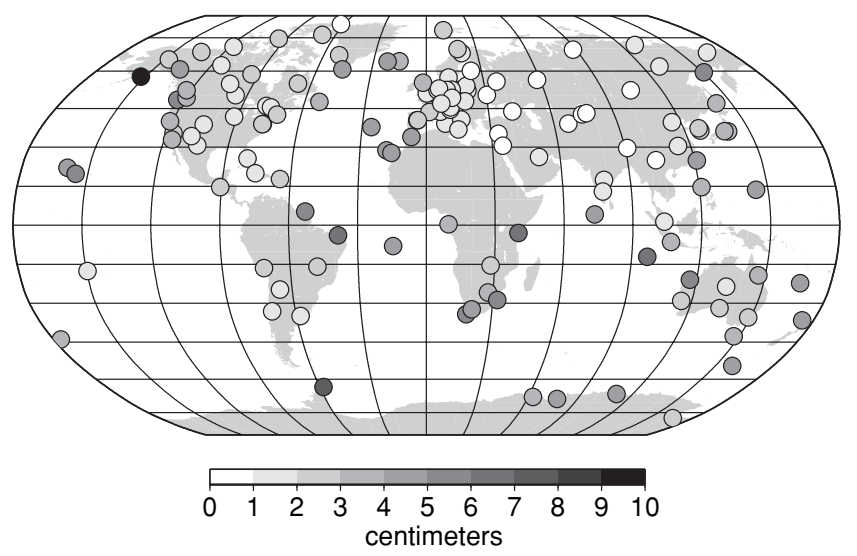

Fig. 1. Maximum magnitudes of the vertical site displacements (in $\mathrm{cm}$ ) due to OTL based on the GOT00.2 ocean tide model

Scherneck 2003) and neglect further tides, as well as nodal modulation.

Figure 1 shows the maximum magnitudes of the vertical site displacements due to OTL, $M^{\mathrm{v}}$, computed from the GOT00.2 ocean tide model by summing up the vertical amplitudes of the 11 main constituents

$M^{\mathrm{V}}=\sum_{i=1}^{11} A_{i}^{\mathrm{v}}$

for each GPS site considered in the data analysis. The resulting magnitudes of the vertical site displacements are of the order of $1-2 \mathrm{~cm}$ in continental regions and they may reach up to $7-9 \mathrm{~cm}$ at the coast. The horizontal displacements due to OTL are approximately a factor of 3-10 smaller than those in the vertical component. However, in reality, taking into account phase relationships, the maximum possible magnitudes of the site displacements are smaller. Summing up the amplitudes of the constituents will always give an over-estimate of the maximum possible magnitude (Yi et al. 2000).

\section{Validation method}

We use the following approach proposed by Dach and Dietrich (2001) for validating OTL models. The geocentric position vector $\mathbf{r}_{\mathbf{0}}(t)$ of a GPS tracking site is corrected by the product of the OTL displacement vector $\mathbf{e}(t)$ (transformed from the local to the geocentric coordinate system) and a scale factor $f$

$\mathbf{r}(t)=\mathbf{r}_{\mathbf{0}}(t)+f \mathbf{e}(t)$

The scale factor $f$ is estimated for each site in a standard least-squares adjustment process of GPS observations simultaneously with all other parameters. The scale factor is used as an indicator of the agreement of the OTL model with the actual site displacements.

An $f$ value close to unity indicates that the model is confirmed by the GPS tracking data. A significant deviation from unity would indicate a model misfit in amplitude, or in phase, or in both, of one or more ocean tide constituents. Using this approach, it is not possible to assign model errors to specific constituents or to decide whether amplitude or phase, or both, show discrepancies. This method is suitable to detect model misfits in general on a global scale using a minimum number of additional parameters.

\section{Data set and analysis}

GPS observations from 138 globally distributed IGS tracking sites, covering a time period of 46 days, from February 09 to March 262003 (day of year 2003: 4085 ), were analyzed. The development version 5.0 of the Bernese GPS Software (Hugentobler et al. 2001) was used for this purpose.

We used double difference GPS carrier-phase measurements performing a complete network solution. An elevation cut-off angle of $3^{\circ}$ was imposed on the solution. Carrier-phase ambiguities were solved and a considerable number were kept fixed to the known integers. Polar tides, solid-Earth tides (see McCarthy 2003), and OTL corrections were applied. Non-tidal loading, such as atmospheric loading, is not considered, following the current practice of GPS analyses in the IGS.

Site coordinates, troposphere parameters, and OTL scale factors were determined in a minimum-constraint network solution for each of the 138 GPS tracking sites. For self-consistency, orbital elements and Earth rotation parameters were also estimated. The datum was defined by adopting three reference frame constraints in terms of no-net-rotation conditions for about 40 carefully selected fiducial sites. Tropospheric delays were modeled by estimating 12 zenith delay parameters for each station per day, using the Niell mapping functions (Niell 1996). In addition, one pair of troposphere gradients was estimated for each station per day to model azimuthal asymmetries (see e.g. Davis et al. 1993).

The analysis was performed using the three abovementioned ocean tide models (FES95.2.1, FES99, GOT00.2). The OTL correction values were computed in March 2003 by Scherneck (2003). Unconstrained daily site-specific OTL scale factors were estimated, together with all mentioned parameters for each solution type.

The individual daily solutions were accumulated at the normal equation level to obtain one combined solution, including the site-specific combination of the OTL scale factors to one set of average factors for the processed interval of 46 days. The site coordinates were also combined into one set of coordinates. The time period of 46 days is large enough to demonstrate the capacity of the validation method. However, a larger time series, optimally more than a year, may help further to decorrelate effects such as non-tidal loading from OTL.

\section{Results}

In this section we analyze the OTL scale factors resulting from the parameter adjustment process. First, we give an overview of the average scale factors estimated for 
the 138 GPS sites and compare the results obtained for the three ocean tide models (Sect. 6.1). Second, we have selected a few sites to demonstrate the behavior of the scale factors in more detail (Sect. 6.2). In Sect. 6.3 we address some problems (length of observation interval, tidal aliasing, correlation effects) affecting the estimation of the scale factors.

\subsection{Scale factors for OTL estimated in a global solution}

The site-specific scale factors for OTL in the combined solution using model GOT00.2 are shown in Fig. 2. The point size indicates the maximum magnitudes of the vertical site displacements, $M^{\mathrm{v}}$, due to OTL (see Fig. 1), decreasing with the distance to the coast. The histogram in Fig. 3 shows the distribution of these average scale factors.

Most scale factors are close to unity, implying that the used OTL model is confirmed on average by the GPS analysis. Most of the factors deviating significantly from unity are found in the inner part of the Eurasian plate. This result is most likely due to the fact that OTL displacements are very small, within the noise level, for inner continental regions, implying that the scale factor

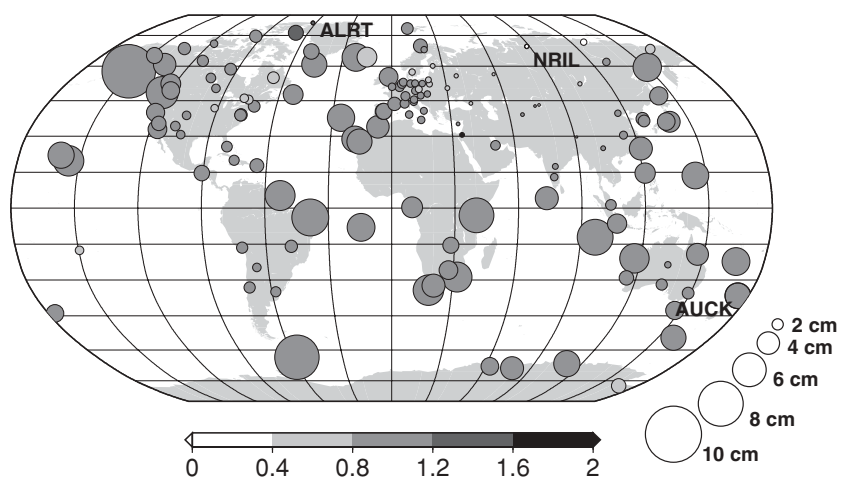

Fig. 2. Average scale factors for OTL using the GOT00.2 ocean tide model. Circle diameters indicate the maximum magnitudes of the vertical site displacements due to OTL

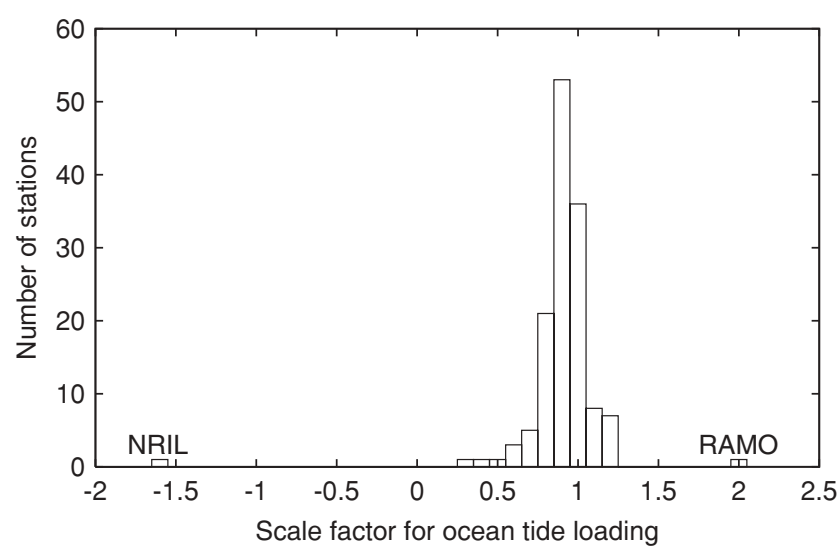

Fig. 3. Distribution of the average scale factors for OTL using the GOT00.2 ocean tide model

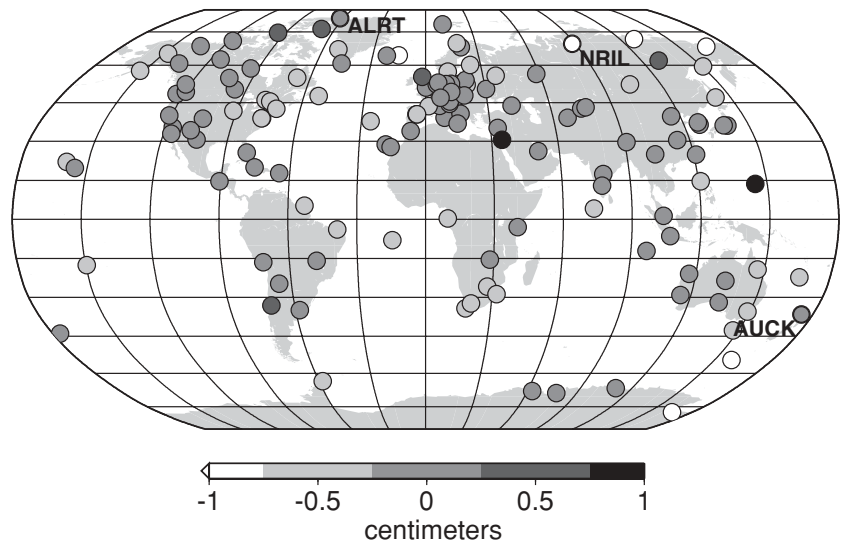

Fig. 4. Corrections of the maximum vertical magnitudes of OTL (in $\mathrm{cm}$ ) derived from the average scale factors using the GOT00.2 ocean tide model

itself is very poorly determined. The minimum value of $f=-1.6$ was estimated for the Russian site Norilsk (NRIL) in northern Eurasia, and the maximum value of $f=2.1$ for the site Mitzpe Ramon (RAMO) in Israel.

Due to the varying size of OTL displacements, it might be more meaningful to express the scale factors in terms of vertical magnitude corrections by multiplying $f-1$ by the maximum magnitude $M^{\mathrm{v}}$

$\Delta e^{\mathrm{v}}=f M^{\mathrm{v}}-M^{\mathrm{v}}$

Such values may be interpreted as model discrepancies. Figure 4 visualizes the corrections $\Delta e^{v}$ estimated for the GOT00.2 ocean tide model. The coastal sites show larger corrections than other sites. The correction values for inner continental sites are close to zero. The extreme values are obtained for NRIL and RAMO, although both sites have relatively small theoretical displacements due to OTL. The discrepancy $\Delta e^{v}$ for the site NRIL is about $-2 \mathrm{~cm}$ and it is about $1 \mathrm{~cm}$ for RAMO. It may be assumed that on-site GPS tracking problems are the cause of the discrepancy at RAMO.

Figure 5 shows the reproducibility (weighted standard deviation) $\sigma$ of the daily estimated scale factors with respect to the average scale factor for GOT00.2. As expected, because of the small OTL effect in the inner part of the Eurasian plate, the biggest standard deviations show up there and underline the difficulty of validating very small OTL signals.

Figure 6 shows the scale factors in units of the dayto-day reproducibility

$f_{r}=\frac{(f-1)}{\sigma}$

Scale factors $f$ with values $-1 \leq f_{r} \leq 1$ are within a onesigma interval around the mean value of unity. Almost all sites obtain values within this range, i.e. the estimated scale factors confirm the used OTL models for most of the GPS sites. Four sites exhibit values exceeding the one-sigma interval (black and white circles in Fig. 6). Three of them are very close to the one-sigma interval boundaries, the exception is for the site NRIL with a value of $f_{r}=-1.5$. 


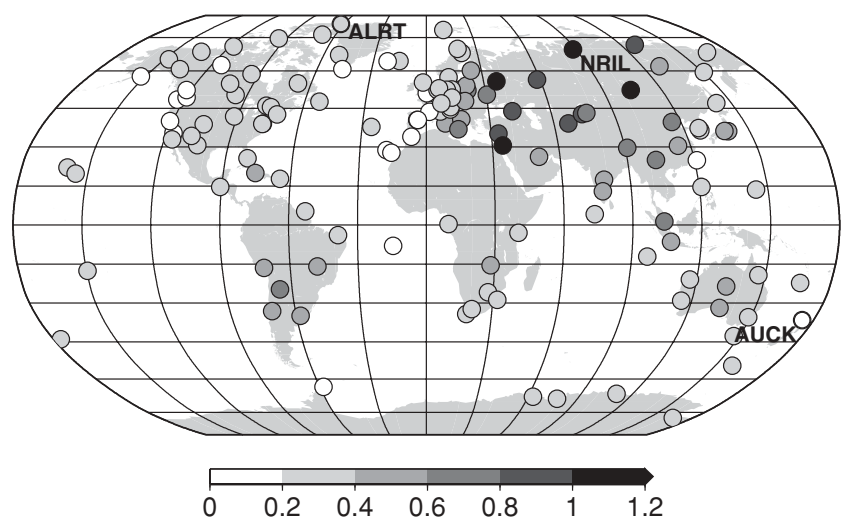

Fig. 5. Reproducibility of the daily estimated scale factors for OTL using the GOT00.2 ocean tide model

Now we compare the results obtained for the GOT00.2 ocean tide model with the results for the FES95.2.1 and FES99 models. Figures 7 and 8 depict the corrections $\Delta e^{v}$ of the vertical site displacement due to OTL derived from the average scale factors using the FES95.2.1 and FES99 models, respectively, relative to the corrections estimated using the GOT00.2 model. The displacement corrections for the OTL models derived from FES95.2.1 (see Fig. 7) differ from those derived from GOT00.2 by up to $1 \mathrm{~cm}$ in the area around the Indonesian archipelago. This might be attributed to the TOPEX/Poseidon data assimilated in the FES95.2.1 ocean tide model. These altimeter data are only fragmentary covering the Indonesian area due to the large density of islands (Daly 2001). The differences between the site displacement corrections for the OTL models derived from the more recent GOT00.2 and FES99 ocean tide models are fairly small (see Fig. 8) and reach a maximum value of about $4 \mathrm{~mm}$ at coastal sites. The repeatabilities of the daily estimated scale factors for the FES95.2.1 and FES99 ocean tide models are quite similar to those achieved with GOT00.2, which is why we do not display them.

\subsection{Scale factors for OTL for selected GPS sites}

Let us now more closely inspect the scale factors for the three GPS sites: Alert (ALRT) in Greenland, Auckland (AUCK) in New Zealand, and Norilsk (NRIL) in northern Eurasia (marked in all previous figures). They are selected more or less arbitrarily, but to have the greatest possible differences in characteristics (different geographical location, different magnitudes in site displacement due to OTL, and different estimated scale factors).

Figures $9 \mathrm{a}, \mathrm{c}$ and $9 \mathrm{e}$ depict the vertical site displacements of each site due to OTL for the processing interval of 46 days and for each of the three models. The different models are indicated by different types of lines, note the differences in scale. The site displacements look very similar for all three models at the sites AUCK and NRIL. They differ slightly in size, by small amounts

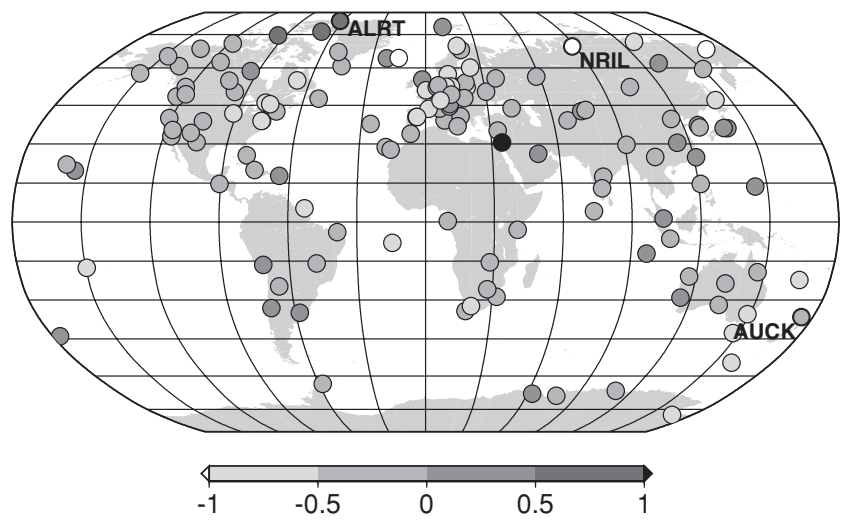

Fig. 6. Average scale factors for OTL using the GOT00.2 ocean tide model expressed in units of the reproducibility

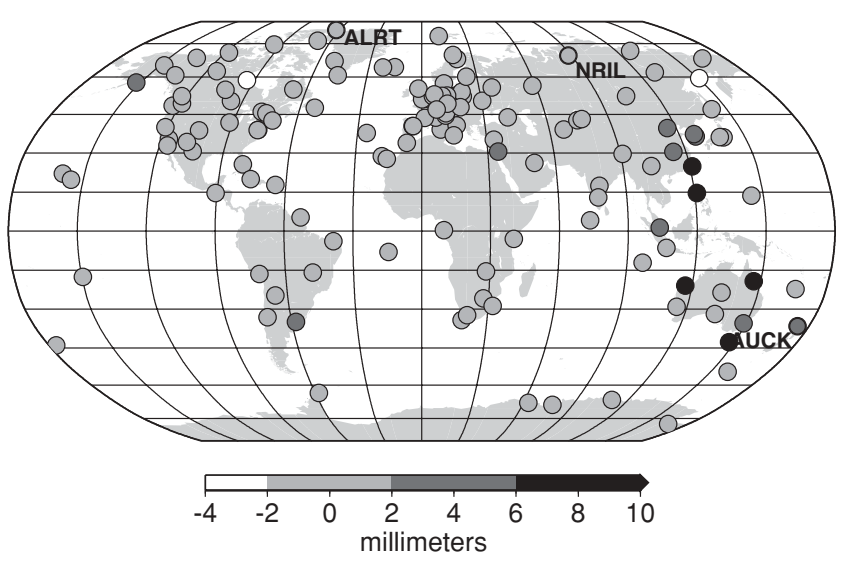

Fig. 7. Corrections of the maximum vertical magnitudes of OTL (in $\mathrm{mm}$ ) derived from the average scale factors using the GOT00.2 ocean tide model relative to the corrections estimated using the FES95.2.1 model

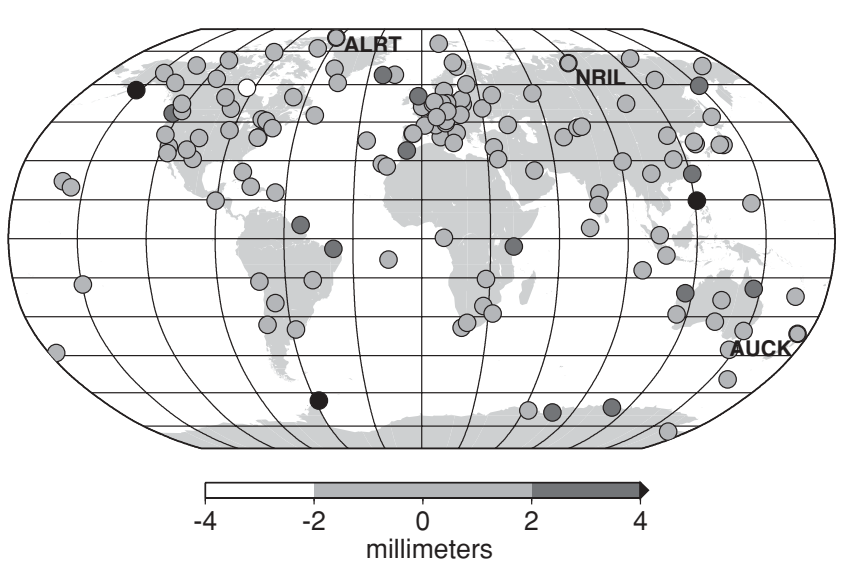

Fig. 8. Corrections of the maximum vertical magnitudes of OTL (in $\mathrm{mm}$ ) derived from the average scale factors using the GOT00.2 ocean tide model relative to the corrections estimated using the FES99 model

compared to the total displacement. For the site ALRT, model differences in phase as well as in magnitude are visible. 


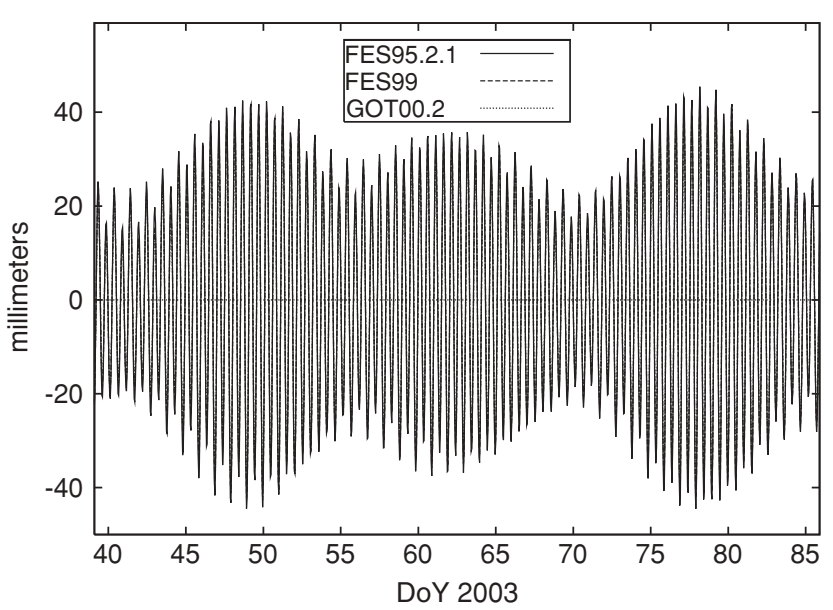

(a) AUCK

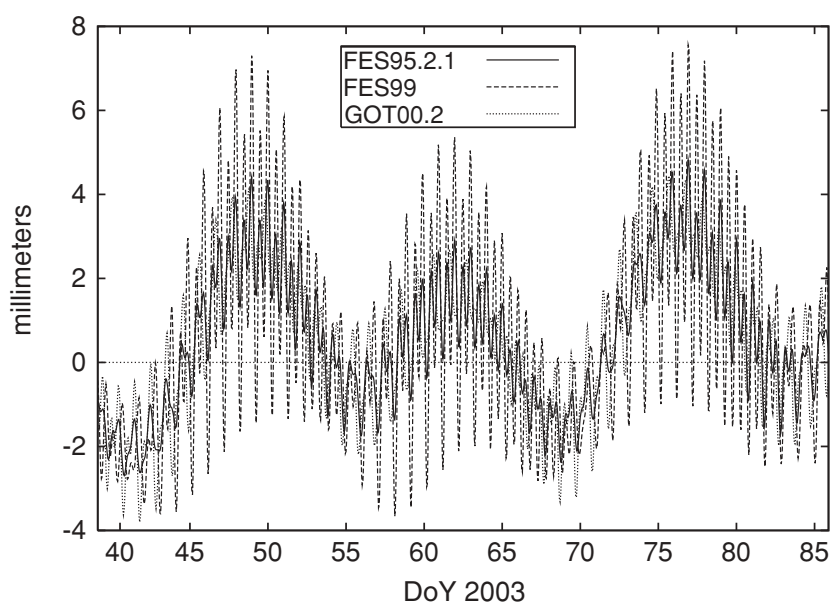

(c) ALRT

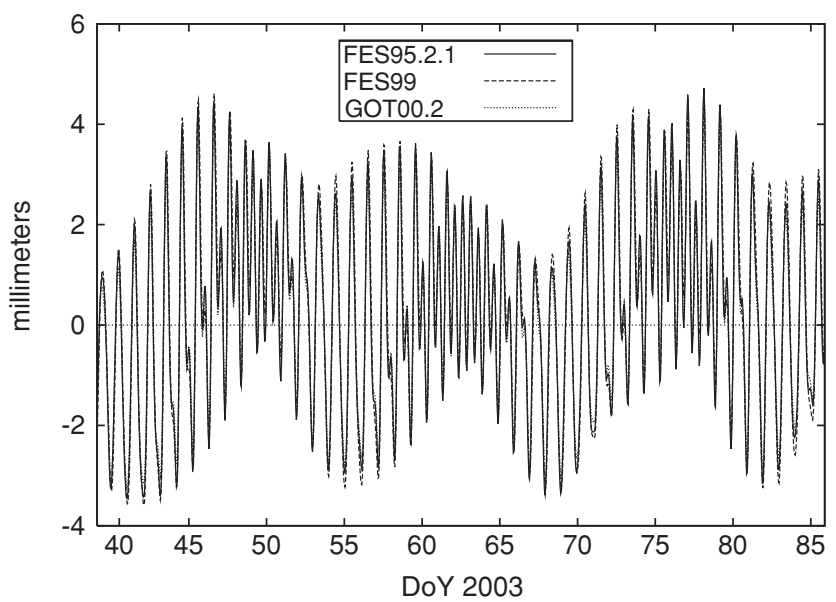

(e) NRIL

Fig. 9. a, c, e Vertical site displacements (in $\mathrm{mm}$ ) due to OTL derived from the FES95.2.1, FES99, and GOT00.2 tide models for each of the sites ALRT, AUCK, and NRIL starting in February 2003. b, d, f Daily site-specific scale factors for OTL with formal RMS errors for

The corresponding daily estimated scale factors for OTL and their formal RMS errors for the AUCK, ALRT, and NRIL sites are shown in Figs. 9b, 9d, and 9f, respectively. The horizontal lines represent the

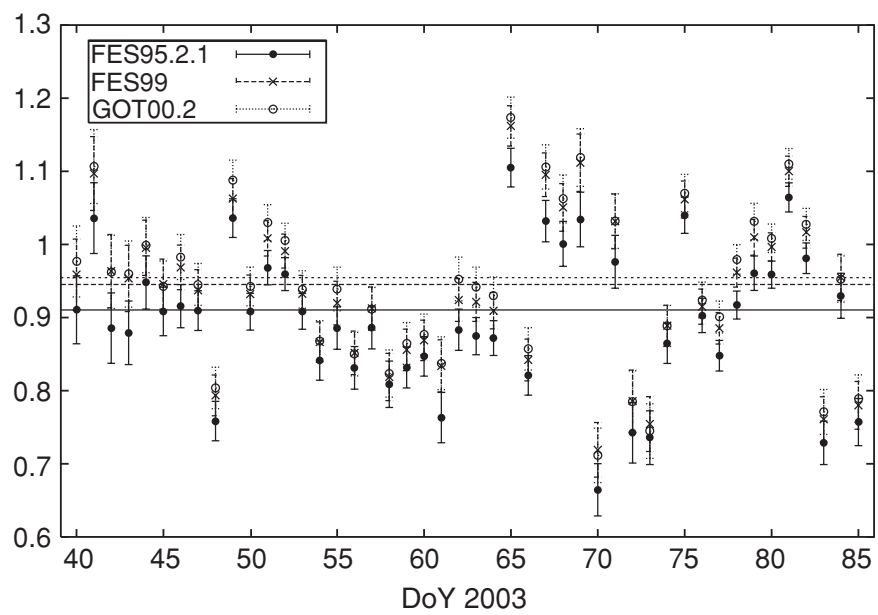

(b) AUCK

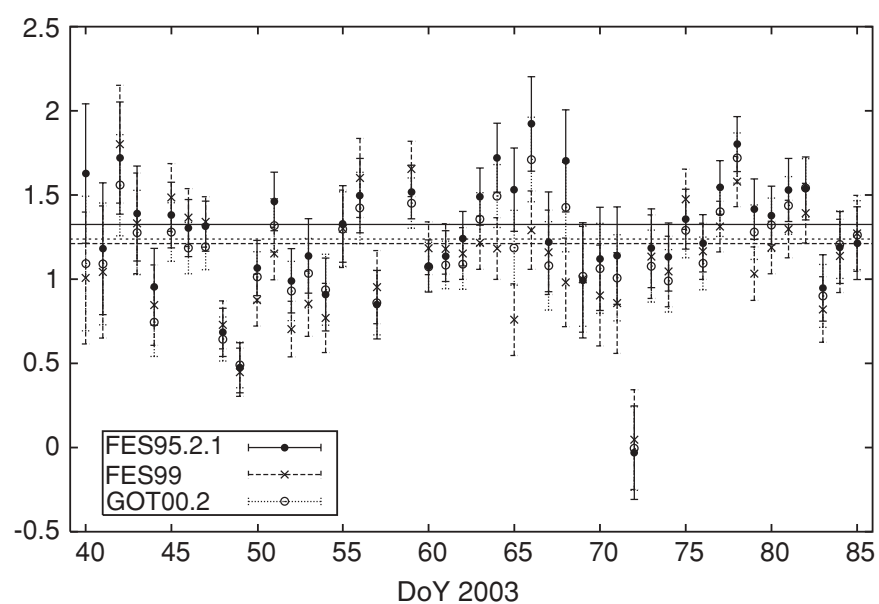

(d) ALRT

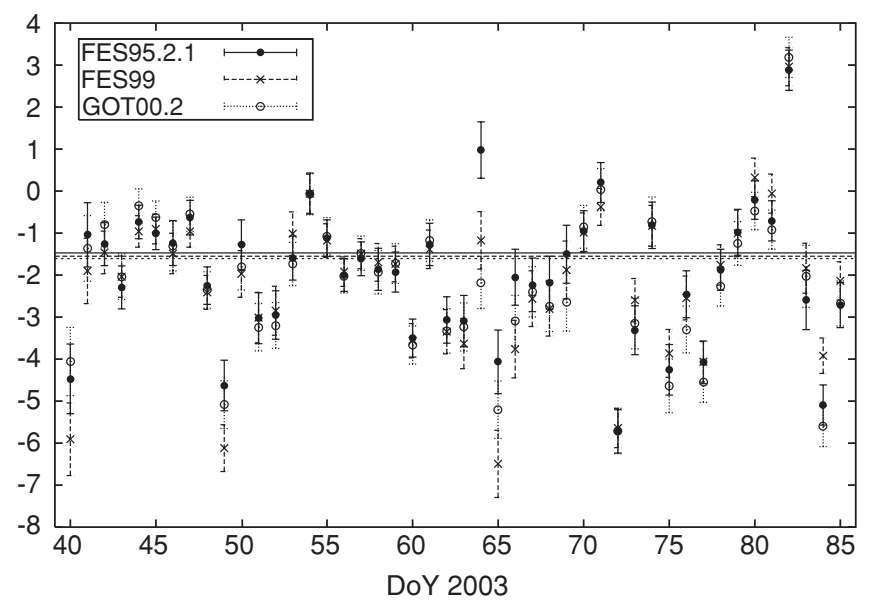

(f) NRIL

the sites ALRT, AUCK, and NRIL starting in February 2003. The horizontal lines represent the average scale factors of the combined solution for each ocean tide model

average scale factors of the combined solutions. The numerical values of the average scale factors $f$, as well as the reproducibility $\sigma$ (weighted RMS of the daily scale factors with respect to the combined solution), are given 
Table 1. Average scale factor $f$, its reproducibility $\sigma$ (weighted RMS value with respect to the combined solution), maximum vertical magnitudes due to ocean tide loading $M^{\mathrm{v}}$ (in $\mathrm{cm}$, from the OTL model), correction of the vertical site displacement $\Delta e^{v}$ de- rived from the average scale factor (in $\mathrm{cm}$ ), average scale factors in units of the reproducibility $f_{r}$ for the GPS sites AUCK, ALRT and NRIL, and the OTL models derived from the FES95.2.1, FES99 and GOT00.2 ocean tide models

\begin{tabular}{|c|c|c|c|c|c|c|}
\hline GPS site & $\begin{array}{l}\text { Ocean tide } \\
\text { model }\end{array}$ & $\begin{array}{l}f \\
{[1]} \\
\end{array}$ & $\begin{array}{l}\sigma \\
{[1]}\end{array}$ & $\begin{array}{l}M^{\mathrm{v}} \\
{[\mathrm{cm}]}\end{array}$ & $\begin{array}{l}\Delta e^{v} \\
{[\mathrm{~cm}]}\end{array}$ & $\begin{array}{l}f_{r} \\
{[\sigma]} \\
\end{array}$ \\
\hline AUCK & FES95.2.1 & 0.91 & \pm 0.092 & 5.13 & -0.46 & -0.98 \\
\hline \multirow[t]{3}{*}{ ALRT } & FES95.2.1 & 1.32 & \pm 0.345 & 0.56 & 0.18 & 0.93 \\
\hline & FES99 & 1.21 & \pm 0.316 & 0.95 & 0.20 & 0.67 \\
\hline & GOT00.2 & 1.24 & \pm 0.314 & 0.62 & 0.15 & 0.76 \\
\hline & GOT00.2 & -1.61 & \pm 1.734 & 0.72 & -1.88 & -1.51 \\
\hline
\end{tabular}

in Table 1 . In addition, the maximum vertical magnitudes of site displacements due to OTL, $M^{\mathrm{v}}$, and the magnitude correction, $\Delta e^{\mathrm{v}}$, derived from the average scale factor are given in $\mathrm{cm}$. The last column in Table 1 gives the scale factor in units of the reproducibility, $f_{r}$.

The GPS site AUCK shows an average OTL scale factor close to unity and a very good reproducibility of about 0.1 for all three models. The scale factors are within the one-sigma interval. The loading signal is highly significant (up to $5 \mathrm{~cm}$; see e.g. Fig. 9a) and therefore the formal RMS error of the estimated factors is very small. The vertical magnitude correction $\Delta e^{\mathrm{v}}$ for the oldest of the three models, FES95.2.1, is, at -4.6 $\mathrm{mm}$, noticeably larger than those for the newer ones $(-2.5 \mathrm{~mm}$ for FES99 and $-2.3 \mathrm{~mm}$ for GOT00.2).

The average loading factors $f$ for the site ALRT are also around unity. The modest reproducibility of about \pm 0.3 can be explained by the OTL effect, which is 10 times smaller than that of the site AUCK (see Fig. 9c). The significant sub-daily differences between the vertical site displacements extracted from the three OTL models do not result in significant differences between the daily estimated scale factors. The factors represent mean values over an interval of one day. Therefore, they are only able to absorb the mean value of unmodeled subdaily variations in site displacements. The corrections $\Delta e^{v}$ for the vertical magnitude derived from the average scale factors for ALRT are smaller than the corrections for AUCK (see Table 1). The scale factors are within the one-sigma interval. We therefore conclude that the used OTL models for the GPS sites AUCK and ALRT are confirmed by the GPS data.

The site displacements for NRIL are very small during the time interval considered (up to $8 \mathrm{~mm}$ from peak to peak; Fig. 9e). Consequently, the resulting scale factors show more pronounced variations, of \pm 1.7 (due to larger formal errors). Nevertheless, the significant offset from 1 of about -2.5 , which is 1.5 times larger than the reproducibility, seems to reveal a modeling problem at this site, which may be due to the site's close proximity to the Arctic Ocean. The Arctic tides depend on hydrodynamic modeling only. Altimeter measurements from TOPEX/Poseidon, which are very important for model accuracy, are not available for that region.
Furthermore, we compared the scale factors of different GPS sites to check whether the scale factor absorbs effects other than OTL, e.g. solid-Earth tides. The effect of solid-Earth tides depends on the longitude and latitude of the GPS site considered. The longitude of the site influences only the timing, i.e. the phase of a constituent (see e.g. Vaníček and Krakiwsky 1986). Therefore, a model error in solid-Earth tides should be the same for all sites located at the same latitude. However, we did not observe such characteristics. The daily estimated scale factors of GPS sites located at the same latitude do not reveal any significant common pattern.

The OTL effect, on the other hand, does not depend on the geographical location but on the distribution of water and land masses on the Earth's surface. Therefore, the OTL effect, as well as model errors, will be the same for sites located close to each other. One example is given in Fig. 10, which shows the scale factors estimated for the sites USUD (Usuda) and TSKB (Tsukuba) in Japan using the GOT00.2 model. The OTL models give

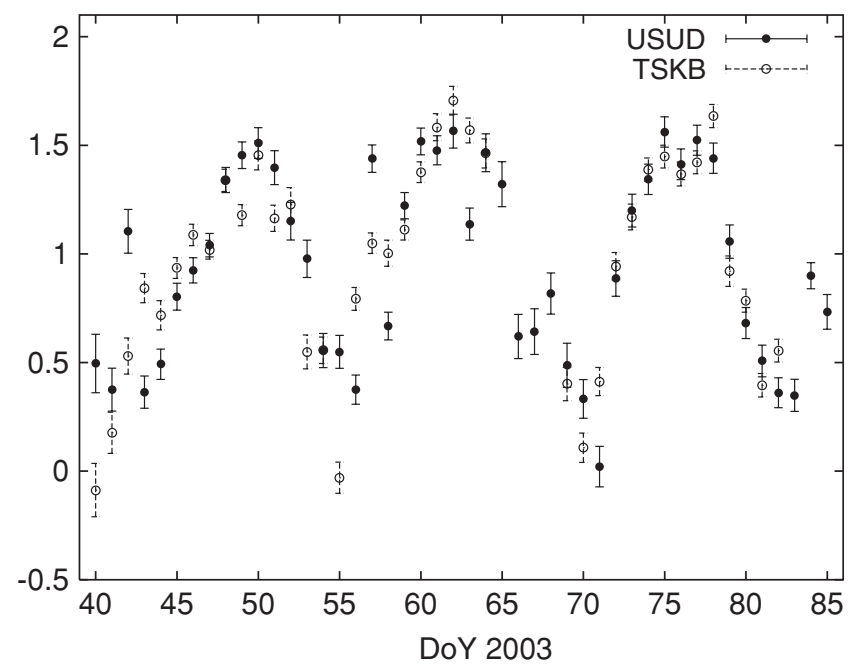

Fig. 10. Daily site-specific scale factors for OTL with formal RMS errors for the sites USUD and TSKB estimated using the GOT00.2 ocean tide model 
roughly the same values for both sites. The maximum vertical magnitude of site displacements due to OTL for TSKB, located on the coast, may reach $1.9 \mathrm{~cm}$. For the site USUD, lying $150 \mathrm{~km}$ inland from the coast, the maximal vertical magnitude reaches $1.6 \mathrm{~cm}$.

We observed a significant common pattern of the daily estimated scale factors for both sites. We found the same effect when comparing other GPS sites located close to each other. We therefore conclude that the scale factors primarily absorb OTL effects. Furthermore, a period of about 15 days could be observed for the scale factors shown in Fig. 10. This period may indicate a modeling problem, e.g. a phase shift in one of the constituents. A phase shift in, for example, $S_{2}$ or $M_{2}$ may result in a period of about 15 days for the daily scale factors due to the beat frequency of $1 / 14.8$ days between these two constituents.

In summary, none of the three OTL models considered here shows significant discrepancies from the predicted OTL effects for most of the 138 processed GPS sites, except for some outliers such as the sites NRIL and RAMO. As expected, the site displacements due to OTL extracted from the recent FES99 and GOT00.2 ocean tide models are slightly better confirmed by the GPS data than are those extracted from the FES95.2.1 model.

\subsection{Several effects on the estimation of the scale factor for OTL}

This section briefly addresses several effects on the estimation of the scale factors, namely effects due to the observation interval, aliasing due to the GPS satellite orbital period, and the correlation with the tropospheric zenith path delay.

Our data analysis is intended to be a first 'quick-look' study rather than an intensive analysis. Therefore, only three OTL are used, and a relatively short observation interval of about seven weeks in February/March 2003 is processed. Due to the superposition of tidal constituents and the resulting beat frequencies of about $1 / 200$ days

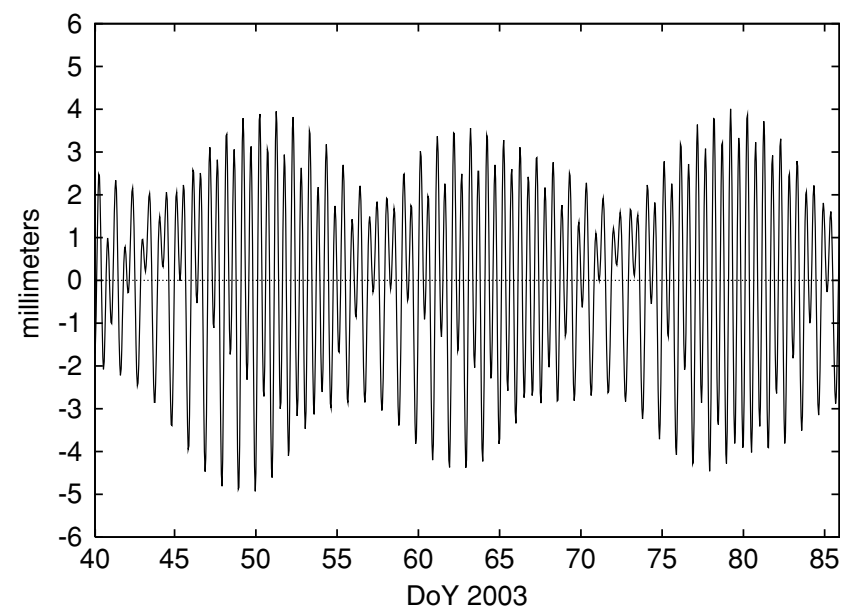

Fig. 11. Differences between the vertical OTL displacements obtained from the FES99 and GOT00.2 ocean tide models for the GPS site ALRT and greater, the scale factors may change depending on the time interval used. For further studies, a much longer time period (e.g. several years) should be considered.

Another aspect to consider is tidal aliasing due to the satellite orbital period (or twice that period). Due to the 2:1 commensurability of the orbital period of the GPS satellites and the sidereal day, the partial tides $K_{1}$ and $K_{2}$ are highly correlated with the orbital period. Therefore, it is, in principle, not possible to derive OTL parameters for these tides from GPS measurements, as, for example, Kirchner (2001) and Schenewerk et al. (2001) have already shown. This situation could be improved by using data from GLONASS satellites in a combined GPSGLONASS data processing, because the revolution period of the GLONASS satellites is about 11 hours 16 minutes, outside the 2:1 commensurability (Rothacher 1998). Also, the new Galileo satellite system may help to distinguish between orbital and tidal effects.

The high correlation of vertical site displacements and tropospheric zenith path delays is well known, and has to be considered for the estimation of OTL parameters (see e.g. Kirchner 2001; Khan Scherneck, 2002; Vey et al. 2002). An unmodeled sub-daily loading effect may seriously affect the estimated troposphere parameters. In our GPS analysis, we solved for site coordinates and 12 troposphere parameters for each site per day. Due to this solution strategy, the tropospheric zenith path delay is capable of partially absorbing OTL effects.

To demonstrate this mechanism, we compared the vertical site displacements attributed to OTL of one station when using two tide models. This is shown in Figs. 11 and 12 for the GPS site ALRT. Figure 11 shows the differences of the ocean loading displacements derived from the FES99 and GOT00.2 ocean tide models. The corresponding differences of the troposphere parameters, shown in Fig. 12, are mainly due to the loading displacement differences. Both figures are highly correlated, indicating that a major part of the unmodeled OTL

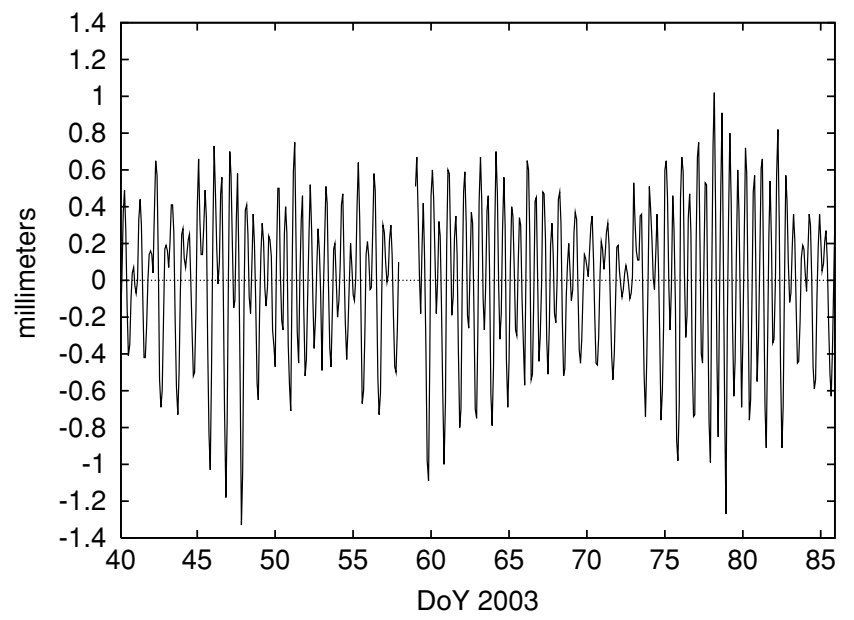

Fig. 12. Differences between the troposphere parameters estimated by introducing the loading displacements derived from the FES99 and GOT00.2 ocean tide models for the GPS site ALRT 


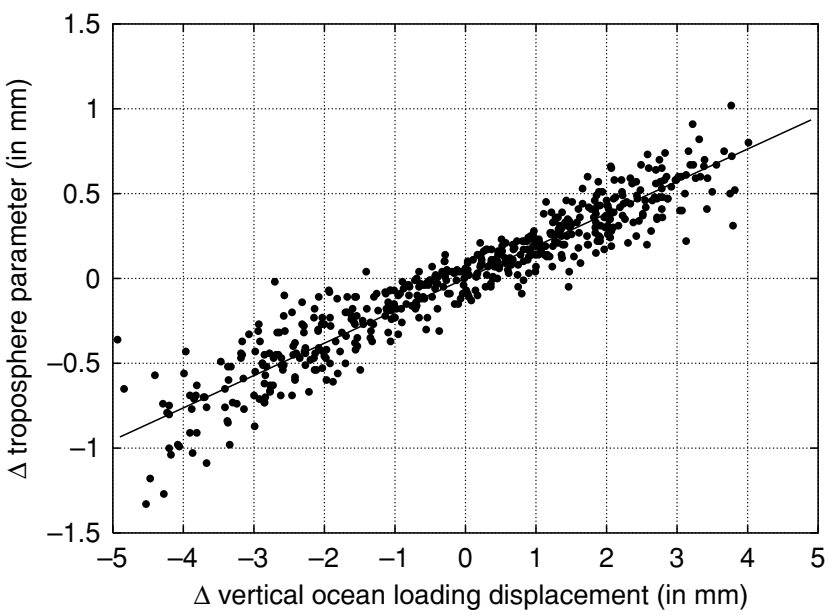

Fig. 13. Correlation diagram of the differences of the vertical site displacements due to OTL and of the troposphere parameters estimated using the FES99 and GOT00.2 tide models for the GPS site ALRT; correlation coefficient $=0.889$

displacements is absorbed by the troposphere parameters. Figure 13 shows the corresponding correlation diagram of vertical site displacements and troposphere parameters for the site ALRT. We obtain a correlation coefficient of 0.889 , which is remarkably high.

The estimated scale factors for OTL displacements are not capable of absorbing modeling errors on a subdaily level. Troposphere parameters with a high temporal resolution will always be affected by unmodeled vertical site displacements.

Although the horizontal displacements due to OTL are small compared to the vertical displacements, a model error in the horizontal plane would be recognizable within the same order of magnitude as an error in the vertical direction due to the characteristics of the GPS: the horizontal site coordinates are estimated much better (by a factor of about three) than the vertical components. Therefore, the horizontal site displacements due to OTL should always also be taken into account in a GPS analysis. The horizontal components are not correlated with the tropospheric zenith path delay. Therefore, a validation of OTL models analyzing the horizontal components separately from the vertical components would make sense.

\section{Conclusions}

The method described for validating global OTL models is well suited for checking the site displacements predicted by these models by means of GPS observations. Site-specific scale factors were estimated for each OTL model. These scale factors allow a validation of OTL models in a general sense. Just one additional parameter for each station has to be estimated, together with all other parameters in one program run. This simple validation method does not, however, allow model discrepancies to be assigned to specific harmonics, or to the amplitude or the phase of a tidal constituent. In order to obtain this information from
GPS observations, all tidal amplitude and phase components for each station have to be estimated.

The results presented show, in general, a good agreement between the FES95.2.1, FES99, and GOT00.2 ocean tide models and the actual site displacements emerging from our GPS analysis. We estimated scaling factors close to unity for most of the 138 globally distributed IGS tracking sites, indicating that the used ocean tide models are confirmed by the GPS data. Factors deviating significantly from the value of unity are found in the inner part of the Eurasian plate, where the OTL effect is very small and the scale factors are poorly determined.

The FES95.2.1 model reveals small discrepancies in the area around the Indonesian archipelago, which might be due to the assimilated TOPEX/Poseidon altimeter data not completely covering the Indonesian area. Parameters correlated with station height and setup with a high time resolution (e.g. tropospheric zenith delays) are affected by unmodeled sub-daily OTL site displacements. Therefore, the use of the best-possible OTL models is essential for zenith path delay estimations and their correct (e.g. meteorological) interpretation.

By estimating a triplet of scale factors for each site, one for each component (north-south, east-west, vertical), it would be possible to validate the horizontal and vertical components of the OTL models separately. Horizontal site displacements, which are not correlated with tropospheric zenith path delays, seem to be well suited to such an investigation.

Our results seem to indicate that an attempt to retrieve GPS-based OTL corrections as part of a general parameter adjustment process could make sense. In principle, it should be possible to estimate site-specific amplitudes and phases for each of the main constituents on the basis of GPS data, except for the tidal waves $K_{1}$ and $K_{2}$ with periods commensurate with the GPS satellites' orbital period (Schenewerk et al. 2001) (a situation that could be improved by using GLONASS or Galileo orbits). Such an analysis would be very interesting from the scientific point of view, but it would also be most demanding in terms of computational resources.

\section{References}

Baker TF, Curtis DJ, Dodson AH (1995) Ocean tide loading and GPS. GPS World 6(3):54-59

Dach R, Dietrich R (2001) The ocean tide loading effect in the GPS analysis: a case study in the Antarctic Peninsula region. Mar Geod 24:13-25

Daly JL (2001) TOPEX-Poseidon radar altimetry: averaging the averages. Available at http://www.john-daly.com/altimetry/ topex.htm

Davis JL, Elgered G, Niell AE, Kuehn CE (1993) Ground-based measurements of gradients in the 'wet' radio refractivity of air. Rad Sci 28(6):1003-1018

Farrell WE (1972) Deformation of the Earth by surface loads. Rev Geophys Space Phys 10(3):761-797

Hugentobler U, Schaer S, Fridez P (2001) Bernese GPS Software version 4.2. Documentation, Astronomical Institute University of Bern 
Khan SA, Scherneck H-G (2002) The $M_{2}$ ocean tide loading wave in Alaska: vertical and horizontal displacements, modeled and observed. J Geod 77(3-4):117-127

Kirchner M (2001) Study of local site displacements due to ocean tide loading using a GPS Network in Island. Res rep 184, Onsala Space Observatory, Onsala

Lambeck K (1988) Geophysical geodesy-The slow deformations of the Earth. Oxford University Press, Oxford

Le Provost C, Genco ML, Lyard FH, Vincent P, Canceil P (1994) Spectroscopy of the world ocean tides from a finite element hydrological model. J Geophys Res 99(C12):2477724798

Le Provost C, Lyard FH, Molines JM, Genco ML, Rabilloud F (1998) A hydrodynamic ocean tide model improved by assimilating a satellite altimeter-derived data set. J Geophys Res 103(C3):5513-5529

Lefèvre F, Lyard FH, Le Provost C, Schrama E (2002) FES99: a tide finite element solution assimilating tide gauge and altimetric information. J Atmos Ocean Tech 19(9):1345-1356

McCarthy DD (2003) IERS Conventions 2000. IERS Technical Note, electronic draft, Central Bureau of IERS, Observatoire de Paris, Paris. Available at http://maia. usno.navy.mil/ conv2000.html

Niell AE (1996) Global mapping functions for the atmosphere delay at radio wavelengths. J Geophys Res 101:3227-3246

Petrov L, Ma C (2002) Study of harmonic site positions determined by VLBI. J Geophys Res 108(B4):2190

Ray RD (1999) A global ocean tide model from TOPEX/Poseidon altimetry: GOT99.2. NASA Tech Memo 209478, Goddard Space Flight Center, Greenbelt, MD
Rothacher M (1998) Recent contributions of GPS to Earth rotation and reference frames. Habilitation, University Press, University of Bern

Schenewerk MS, Marshall J, Dillinger W (2001) Vertical oceanloading deformation derived from a global GPS network. J Geod Soc Jpn 47(1):237-242

Scherneck H-G (1991) A parameterized solid Earth tide model and ocean tide loading effects for global geodetic baseline measurements. Geophys J Int 106(3):677-694

Scherneck H-G (2003) Automatic service provider. Available at http://www.oso.chalmers.se/ loading

Seidelmann K (1992) Explanatory supplement to the astronomical almanac. University Science Books, Mill Valley, CA

Shum CK, Woodworth PL, Andersen OB, Egbert GD, Francis O, King C, Klosko SM, Le Provost C, Li X, Molines J-M, Parke ME, Ray RD, Schlax MG, Stammer D, Tierney CC, Vincent P, Wunsch CI (1997) Accuracy assessment of recent ocean tide models. J Geophys Res 102(C11):25 173-25 194

Vaníček P, Krakiwsky EJ (1986) Geodesy: the concepts, 2nd edn. Elsevier, Amsterdam

Vey S, Calais E, Llubes M, Florsch N, Woppelmann G, Hinderer J, Amalvict M, Lalancette MF, Simon B, Duquenne F, Haase JS (2002) GPS measurements of ocean loading and its impact on zenith tropospheric delay estimates: a case study in Brittany, France. J Geod 76(8):419-427

Wessel P, Smith WHF (2004) The Generic Mapping Tools (GMT). Available at http://gmt.soest.hawaii.edu

Yi D, Minster B, Bentley CR (2000) The effect of ocean tidal loading on satellite altimetry over Antarctica. Antarc Sci 12(1):119-124 